\title{
Parafoveal information is not sufficient to produce semantic or visual priming
}

\author{
KENNETH R. PAAP and SANDRA L. NEWSOME \\ New Mexico State University, Las Cruces, New Mexico 88003
}

\begin{abstract}
Marcel (1978) has shown that semantic priming can occur in the lexical-decision task even if the prime is masked to the point at which its presence cannot be detected. The purpose of the present experiments was to determine if primes that begin four or five spaces to the right of fixation can also produce semantic facilitation even though they are very difficult to recognize. Experiment 1 showed that facilitation did occur when the parafoveal primes used in the subsequent experiments were presented foveally. In Experiment 2, the primes were moved to the parafovea, but the task demands directed the subject's attention toward the fovea. When subjects were ignoring the information presented to the parafovea (an allocation pattern that should correspond to that used during normal reading), neutral primes were just as effective as either semantically related or identical primes. In Experiment 3, the task demands were altered so that subjects were actively attending to the parafoveal primes. Although subjects were trying to process the primes, there was still no evidence that benefits could be derived from parafoveal primes. A final experiment showed that subjects given extensive practice with the materials will produce large amounts of identity and semantic priming. The results support the conclusion that readers can benefit little from the preprocessing of information in the parafovea unless that information can be supplemented with contextual expectations.
\end{abstract}

Consider a reader who encounters the following sentence without any prior context: "For example, some words are much more predictable than others." If the reader successively fixates to the positions indicated by the boldface type, it can be seen that information in the parafovea about the immediately upcoming word will sometimes be only minimally constrained by the previous context (e.g., the word "words"), but that other words (e.g., the word "others") will be highly predictable. The initial experiments of the present report concern the extent to which unanticipated words in the parafovea are processed and, equally important, whether or not any preprocessing of the parafoveal information actually speeds the reading process when an eye movement brings that part of the text into the fovea. We have tested these basic issues using a variation of the lexical-decision task to determine if a parafoveal prime can facilitate the processing of a related target

Portions of these data were presented at the meeting of the Rocky Mountain Psychological Association, Tucson, April 1980. We would like to thank Susan Jones for collecting most of the data for Experiment 1 and for serving as one of our long-term subjects in Experiment 4. Earlier versions of this paper benefited from the comments of Keith Rayner and the CIP group at NMSU. We are especially indebted to Roger Schvaneveldt for the use of his equipment, computer programs, and general lore for doing lexical-decision experiments. Requests for reprints should be sent to Kenneth R. Paap, Department of Psychology, Box 3452, New Mexico State University, Las Cruces, New Mexico 88003. that is subsequently presented to the fovea. If subjects cannot take advantage of parafoveal information when engaged in a simple lexical-decision task, we would be prepared to argue that they are unlikely to do so when they are confronted by a similar, but more complex, situation during normal reading.

The lexical-decision task requires subjects to indicate, as quickly as possible, whether a letter string is a word or a nonword. Lexical decisions are faster when a target (e.g., NURSE) is preceded by a semantically related prime (e.g., DOCTOR) than when by an unrelated prime (e.g., BUTTER). One inference that follows from this semantic relatedness effect is that the activation of the prime's representation in memory must somehow enhance the accessibility of the target's representation. Marcel (1978) has argued that both the activation of the prime and its ability to enhance the accessibility of related words (the set of semantically related words would include the prime word itself) can occur, even if the prime has not been consciously recognized. This conclusion is based on his report that the amount of semantic facilitation produced in the lexical-decision task does not change when the prime is masked to the point at which each individual subject is no longer able to detect the presence of a word above chance. More specifically, targets preceded by semantically related primes were judged $56 \mathrm{msec}$ faster than those preceded by unrelated primes when no mask was used, compared with a 62-msec difference when the mask was used. 
Since severe conditions of backward masking do not occur during normal reading, one wonders what constitutes the ecological genesis for our ability to prime the lexicon on the basis of information of which we were never consciously aware. One possibility is that Marcel's unconscious priming may be related to the processing of that portion of the upcoming text that is in advance of both the subject's eyes and his conscious awareness. Since the interfixation distance of a skilled reader is approximately a word or two, it is often the case that the word that will be recognized in the next fixation will be in the parafovea during the current fixation. Marcel's results suggest that the word's presence in the parafovea may unconsciously prime its lexical representation and enhance its accessibility. Accordingly, the initial motivation for this study was to determine if primes that begin five spaces to the right of fixation can produce semantic facilitation even though they are difficult to recognize.

The empirical basis for hypothesizing that parafoveal words may produce semantic facilitation was Rayner, McConkie, and Ehrlich's (1978) demonstration that a semantic mismatch between a parafoveal prime and a foveal target can sometimes detract from the amount of the facilitation that accrues from various types of visual similarity between the prime and target. In the standard condition, a word or letter string initially appeared in parafoveal vision, followed by the subject's eye movement to the stimulus. During the saccade, the initial stimulus was replaced by a word that the subject was asked to pronounce. A second condition, called the simulation condition, was designed to investigate whether the facilitation effects are contingent upon the subject's actually making an eye movement. In the simulation condition, the subject maintained fixation on a very dim target dot while a parafoveal stimulus was presented for $190 \mathrm{msec}$. The target word appeared $10 \mathrm{msec}$ after the offset of the parafoveal stimulus and was centered about the position of the fixation dot. As in the standard condition, the subject's task was to name the word as rapidly as possible.

Five types of parafoveal stimuli were used. The prime could be identical to the target word (chestchest), a word that began and ended with the same letters and also maintained the same overall word shape (chart-chest), a nonword with the same external letters and overall shape (chovt-chest), a nonword with the same external letters but a different shape (chfbt-chest), or a nonword with different external letters but the same overall shape (ekovf-chest). In addition, one of the three subjects participated in a replication in which the last prime described was replaced by a single asterisk. The parafoveal primes were presented 1,3 , or $5 \mathrm{deg}$ to the left or right of fixation.
The nature of the parafoveal stimulus interacted with the degree of eccentricity to determine the amount of facilitation. The pattern of interaction observed in the simulation condition was very similar to that obtained in the standard condition. When the primes were only $1 \mathrm{deg}$ from the fovea, the five prime types yielded a consistent order of naming times. Identity primes were the fastest. Primes that had the same external letters and the same overall shape were the next fastest, but nonword primes of this type were faster than word primes, suggesting that a semantic mismatch will detract from the amount of facilitation that is usually produced by a visually similar prime. However, these visually similar word primes were still faster than nonwords that had the same external letters but different shapes. Finally, nonword primes that had the same shape but different external letters consistently produced the slowest naming times. The same order was observed at $3 \mathrm{deg}$ of eccentricity, but the differences between prime types were much smaller and less consistent. At $5 \mathrm{deg}$, the nonwords with the same shapes but different external letters still produced significantly longer naming times than did identity primes, but no other significant differences were observed. The investigators concluded that differences between the prime types reflect different levels of benefit, since the asterisk control produced slower naming times relative to all of the letter-string primes.

On the basis of these data, it appears that when a parafoveal stimulus is presented within 1 to $3 \mathrm{deg}$ of the fovea, visual information concerning individual letters and overall shape can be acquired and used to reduce the amount of visual processing of similar stimuli that are subsequently presented to the fovea. More recently, Rayner, McConkie, and Zola (1980) have used the same paradigm to determine that it is the identity of the first two letters of the parafoveal prime that is particularly effective in producing parafoveal priming.

Although the evidence is certainly weaker, it also seems to be the case that part of the facilitation that accrues from primes that have the same external letters and shape can be attenuated if the prime spells a word that is semantically different from the visually similar target. The interference produced by a lexical mismatch (i.e., the fact that chart-chest produces less benefit than chovt-chest) suggests that primes presented about $1 \mathrm{deg}$ to the right of fixation are often subjected to semantic preprocessing. This lexical effect, especially in the simulation condition, appears to be quite strong for primes 1 deg to the right of fixation, but weak or absent for words either to the left or farther than 3 deg to the right.

One might expect parafoveal primes to produce facilitation, rather than interference, if two of the relationships between Rayner, McConkie, and Ehrlich's 
word primes and targets are altered. First, facilitation may occur if the word prime is semantically related (rather than unrelated) to the target. Second, it may be necessary to remove the visual similarity between the word primes and targets. Both of these modifications were included in Experiments 4 and 5 of the Rayner et al. (1980) report. However, naming time for the semantically related primes did not differ from a semantically unrelated prime that had the same last-two letters as the target. Although these findings should attenuate one's expectation that parafoveal information may be processed to a semantic level and produce facilitation, two characteristics of these experiments make further tests of the hypothesis worthwhile. First, there was no neutral prime, since all prime types had some relationship to the target. Thus, the semantically related primes may have been producing some unmeasured benefits. Second, the semantically related primes were tested in the standard condition in which the semantic interference observed in the earlier studies is consistent but always very small. The present studies used an appropriate neutral prime that would enhance the detectability of small, but reliable, benefits of semantic preprocessing. If semantic facilitation occurs, this would be consistent with the view that upcoming words in the parafovea can unconsciously prime their lexical representations and facilitate word recognition.

\section{EXPERIMENT 1}

The purpose of the first experiment was to determine a baseline of facilitation for the specific materials and types of primes that will be used in later experiments that place the prime in the parafovea rather than the fovea. Since the subsequent experiments will manipulate the locus of the subject's attention by either requiring or not requiring the subject to make an overt response to the prime, the first experiment will also systematically explore the effects of responding vs. not responding to the prime.

\section{Method}

\section{Design}

There were three different groups of subjects, distinguished by their response to the primes. Within each group of subjects, there were four types of primes (semantically related, identical, visually similar, and neutral) and two types of targets (words and nonwords). The combination of the latter two variables produced eight within-subjects conditions. The stimulus materials were counterbalanced in such a way that every word and nonword target appeared in all conditions across subjects. Each subject saw each string of letters only once.

\section{Subjects}

Three groups of 24 subjects participated in order to partially fulfill a requirement for an introductory psychology class. The subjects whose error rates exceeded .06 were replaced.

\section{Stimuli}

Stimulus events and timing operations were controlled by a Cromemco Z80 microcomputer system. The Cromemco Dazzler graphics were used to generate the stimulus characters as a matrix of dots. Patterns for lowercase characters that most closely resembled the lowercase characters shown by Geyer (1977) were selected. The letters selected had a maximum height of $8 \mathrm{~mm}$ and maximum width of $5 \mathrm{~mm}$. The subjects were seated approximately $122 \mathrm{~cm}$ from a Hitachi 9-in video monitor. Most letters subtended a width of about $.23 \mathrm{deg}$. A four-letter string subtended about $1.21 \mathrm{deg}$ and a five-letter string about $1.53 \mathrm{deg}$.

All primes and targets consisted of four or five letters. Onehundred-and-sixty words were selected as target letter strings. Eighty of these words were transformed into nonwords by replacing one letter. Semantically related primes were words that, in the judgment of the experimenters, were related in meaning to the word targets (e.g., nest-eggs). Identical primes were identical to the target words (e.g., eggs-eggs). Visually similar primes were nonwords that had the same shape or envelope as the word targets. Selection of the letters for the visually similar primes was constrained in two ways. First, overall word shape was preserved by always substituting ascenders for ascenders and descenders for descenders. Second, each letter was replaced by the psychophysically most confusable letter as defined by Geyer's (1977) lowercase confusion norms. For example, for the target word eggs, the visually similar prime was oqqn.

The neutral primes consisted of a row of uppercase Xs (e.g., $X X X X$-eggs) and were used to determine whether the effects of the three other prime types should be attributed to costs or benefits (Neely, 1976; Posner \& Snyder, 1975). The number of Xs in the neutral primes was either four or five, depending on the number of letters in the target.

The neutral, identical, and visually similar primes were constructed in the same manner for nonword targets. There were no semantically related primes for the nonword targets. The fourth type of prime for nonword targets consisted of an unrelated fouror five-letter word (e.g., tail-hacp).

\section{Procedure}

The experimental session consisted of a practice block of 32 trials and 5 blocks of experimental trials. Presentation of the primetarget combinations was randomized within each block, with the constraint that four samples of each condition appeared in each block of 32 trials. A trial was initiated by an asterisk that served as both a warning signal and a fixation point. The asterisk remained on the screen for $1 \mathrm{sec}$ and was positioned so that it would be centered between the middle two letters of a four-letter prime or target. The asterisk was immediately followed by a prime that remained on the screen for $133 \mathrm{msec}$. Targets appeared in the same spatial location as the prime and remained in view until the subject responded. The right-hand key was correct for word targets, and the left-hand key was correct for nonword targets.

In Group 1, the subjects were instructed to make no response to the primes. Presentation of the prime in this group was followed by a 500-msec delay during which the screen was blank. Stimulusonset asynchrony (SOA) between the target and the prime was $633 \mathrm{msec}$. This SOA approximates the SOA in the other two groups, since average lexical-decision time was about $630 \mathrm{msec}$.

The subjects in Groups 2 and 3 were instructed to make a lexical decision about both the prime and the target. The subjects in Group 2 were told to respond "no" to the Xs, whereas the subjects in Group 3 were told to respond "yes" to Xs. During the interval between the offset of the prime and the subject's response, the screen remained blank. A correct response to the prime initiated the onset of the target letter string. As was true for Group 1, the word $E R R O R$ appeared on the screen when the subject made an incorrect response to the target letter string. An error message also appeared on the screen when the subject made an incorrect response to the prime. If the subject made a mistake on the prime, the target was not presented. 


\section{Results and Discussion}

Mean response times to the target (excluding incorrect responses) were submitted to an analysis of variance, treating prime response (overt-"yes"' to Xs, overt-"no" to Xs, or no overt response) as a betweensubjects variable and prime type (identical, semantically related, visually similar, or neutral) and target type (word or nonword) as within-subjects variables. The combination of the two within-subjects variables produced eight basic conditions for each group of subjects. For all groups, the subjects judged different pairs of letter strings in each condition. Thus, the interaction of subjects and conditions in the analysis of variance contains variability due to the Item by Condition interactions, as well as variability due to the main effect of items. By using the Subject by Condition interactions as error terms for testing the effects of conditions, we may generalize our conclusions to both the population of subjects and the population of items from which our samples were drawn (Clark, 1973; Schvaneveldt \& McDonald, in press).

In the analysis of reaction times, there were significant main effects of target type $[\mathrm{F}(1,69)=85.68$, $\mathrm{p}<.001]$ and prime type $[\mathrm{F}(3,207)=96.72, \mathrm{p}<.001]$ and a significant Target Type by Prime Type interaction $[F(3,207)=22.69, \mathrm{p}<.001]$. Most important is the significant three-way Target Type by Prime Type by Prime Response interaction $[F(6,207)=6.79$, $\mathrm{p}<.001$ ] that is shown in Table 1 and is discussed in the following sections.

\section{Semantically Related Primes}

The primary purpose of the first experiment was to determine if the particular materials to be used in the subsequent experiments produced typical levels of semantic facilitation when presented foveally. When subjects were not required to respond to the prime (Group 1), word targets preceded by semantically related primes were judged $35 \mathrm{msec}$ faster than those preceded by the neutral primes $[\mathrm{t}(23)=2.40$, $p<.05]$. The obtained benefit of $35 \mathrm{msec}$ is somewhat smaller than the $72-\mathrm{msec}$ benefit recently reported by Schvaneveldt \& McDonald (in press), but is comparable to the $38-\mathrm{msec}$ benefit reported by Neely (1976) and 34-msec benefit reported by Antos (1979). In summary, when our primes are presented foveally, significant benefits accrue that are comparable in size to those frequently reported in the literature.

In the third experiment, the subject's attention will be locked on a parafoveal prime by requiring the subject to make a lexical decision about that prime before the target is presented. Having to make a deliberate and overt response to the prime may alter the subject's strategies or introduce response sequencing biases that, in turn, may affect the pattern of costs and benefits for the various prime types.

These possibilities were tested by requiring Group 2 to make a lexical decision about both the prime and the target. The subjects were instructed to treat the string of Xs as a nonword and, therefore, to respond "no" when the neutral prime appeared. Having to make an overt response to the prime did not intro-

Table 1

Lexical Decision Times (RT) in Milliseconds and Error Rates (ER) to Word (W) and Nonword (NW) Targets for the Four Types of Prime Stimuli

\begin{tabular}{|c|c|c|c|c|c|c|c|c|c|}
\hline & \multirow[b]{3}{*}{ Target } & \multicolumn{8}{|c|}{ Prime Type } \\
\hline & & \multicolumn{2}{|c|}{ Semantically Related } & \multicolumn{2}{|c|}{ Visually Similar } & \multicolumn{2}{|c|}{ Identical } & \multicolumn{2}{|c|}{ Neutral Xs } \\
\hline & & RT & ER & RT & ER & RT & ER & RT & ER \\
\hline & & \multicolumn{8}{|c|}{ Experiment 1} \\
\hline Group 1 & $\begin{array}{l}\text { W } \\
\text { NW }\end{array}$ & $\begin{array}{l}571 \\
661\end{array}$ & $\begin{array}{l}.01 \\
.02\end{array}$ & $\begin{array}{l}604 \\
685\end{array}$ & $\begin{array}{l}.04 \\
.05\end{array}$ & $\begin{array}{l}477 \\
571\end{array}$ & $\begin{array}{l}.01 \\
.04\end{array}$ & $\begin{array}{l}606 \\
678\end{array}$ & $\begin{array}{l}.02 \\
.05\end{array}$ \\
\hline Group 2 & $\begin{array}{l}\text { W } \\
\text { NW }\end{array}$ & $\begin{array}{l}543 \\
719\end{array}$ & $\begin{array}{l}.03 \\
.15\end{array}$ & $\begin{array}{l}635 \\
671\end{array}$ & $\begin{array}{l}.06 \\
.07\end{array}$ & $\begin{array}{l}530 \\
572\end{array}$ & $\begin{array}{l}.00 \\
.01\end{array}$ & $\begin{array}{l}596 \\
743\end{array}$ & $\begin{array}{l}.04 \\
.14\end{array}$ \\
\hline \multirow[t]{5}{*}{ Group 3} & $\begin{array}{l}\text { W } \\
\text { NW }\end{array}$ & $\begin{array}{l}513 \\
712\end{array}$ & $\begin{array}{l}.02 \\
.09\end{array}$ & $\begin{array}{l}608 \\
696\end{array}$ & $\begin{array}{l}.05 \\
.07\end{array}$ & $\begin{array}{l}478 \\
527\end{array}$ & $\begin{array}{l}.00 \\
.00\end{array}$ & $\begin{array}{l}544 \\
721\end{array}$ & $\begin{array}{l}.02 \\
.07\end{array}$ \\
\hline & & \multicolumn{8}{|c|}{ Experiment 2} \\
\hline & $\begin{array}{l}\text { W } \\
\text { NW }\end{array}$ & $\begin{array}{l}557 \\
620\end{array}$ & $\begin{array}{l}.04 \\
.06\end{array}$ & $\begin{array}{l}563 \\
628\end{array}$ & $\begin{array}{l}.04 \\
.06\end{array}$ & $\begin{array}{l}559 \\
614\end{array}$ & $\begin{array}{l}.03 \\
.05\end{array}$ & $\begin{array}{l}551 \\
617\end{array}$ & $\begin{array}{l}.03 \\
.06\end{array}$ \\
\hline & & \multicolumn{8}{|c|}{ Experiment 3} \\
\hline & $\begin{array}{l}\text { W } \\
\text { NW }\end{array}$ & $\begin{array}{l}679 \\
761\end{array}$ & $\begin{array}{l}.02 \\
.04\end{array}$ & $\begin{array}{l}677 \\
779\end{array}$ & $\begin{array}{l}.02 \\
.04\end{array}$ & $\begin{array}{l}646 \\
784\end{array}$ & $\begin{array}{l}.03 \\
.05\end{array}$ & $\begin{array}{l}641 \\
778\end{array}$ & $\begin{array}{l}.01 \\
.06\end{array}$ \\
\hline & & \multicolumn{8}{|c|}{ Experiment 4} \\
\hline Subject S.J. & $\begin{array}{l}\text { W } \\
\text { NW }\end{array}$ & $\begin{array}{l}427 \\
794\end{array}$ & $\begin{array}{l}.00 \\
.06\end{array}$ & $\begin{array}{l}698 \\
686\end{array}$ & $\begin{array}{l}.04 \\
.02\end{array}$ & $\begin{array}{l}294 \\
392\end{array}$ & $\begin{array}{l}.00 \\
.01\end{array}$ & $\begin{array}{l}660 \\
723\end{array}$ & $\begin{array}{l}.01 \\
.01\end{array}$ \\
\hline Subject S.N. & $\begin{array}{l}\text { W } \\
\text { NW }\end{array}$ & $\begin{array}{l}416 \\
498\end{array}$ & $\begin{array}{l}.02 \\
.01\end{array}$ & $\begin{array}{l}549 \\
506\end{array}$ & $\begin{array}{l}.03 \\
.01\end{array}$ & $\begin{array}{l}363 \\
383\end{array}$ & $\begin{array}{l}.00 \\
.00\end{array}$ & $\begin{array}{l}527 \\
557\end{array}$ & $\begin{array}{l}.01 \\
.02\end{array}$ \\
\hline
\end{tabular}


duce any qualitative changes in semantic priming. Word targets preceded by semantically related primes were judged $53 \mathrm{msec}$ faster than those preceded by the neutral primes $[t(23)=5.63, p<.001]$. These benefits were expected, since Meyer, Schvaneveldt, and Ruddy (1975) also observed an effect of semantic relatedness when subjects were required to make successive lexical decisions about both the prime and the target. They report about 38 -msec differences between related and unrelated primes for intact targets and $71-\mathrm{msec}$ differences for degraded targets. Since the differences between related and unrelated primes reported by Meyer et al, reflect the sum of both costs and benefits, it is clear that the $53-\mathrm{msec}$ benefit obtained in our study can be viewed as a fairly large effect.

Since all of the semantically related primes were words, this condition requires two consecutive "yes" responses. Consequently, the same response sequence can be achieved in a control condition by having subjects respond "yes" to the neutral primes. With this control (Group 3), it is still the case that word targets preceded by semantically related primes are significantly faster $(31 \mathrm{msec})$ than those preceded by the neutral primes $[t(23)=2.72, p<.02]$. This result removes any concern that the semantic facilitation observed in Group 2 was an artifact of the "yes"." "yes" sequence that was required for word targets preceded by semantically related primes, compared with the "no"-"yes" sequence that was required in the neutral control.

\section{Visually Similar Primes}

In the absence of any overt response to the prime, neither word nor nonword targets preceded by visually similar primes were significantly faster than those preceded by the neutral primes. This suggests that neither featural similarity nor overall similarity in shape can automatically facilitate the processing of a subsequent word target.

Since the visually similar primes were always nonwords, the correct response-sequencing control for both word and nonword targets requires a "no" response to the neutral-X primes (Group 2). In marked contrast with the results obtained when subjects were not required to respond to the prime, the visually similar primes in Group 2 produced $39 \mathrm{msec}$ of costs for word targets [t(23) $=4.47, \mathrm{p}<.001]$ and 72 msec of benefits for nonword targets $[t(23)=7.34$, $\mathrm{p}<.0011$. It appears to be the case that when the target's lexical status is the same as the prime's and, therefore, requires the same overt response, visual similarity will facilitate the lexical decision; but when the target's lexical status is different from that of the prime and, therefore, requires the opposite response, visual similarity will interfere with the lexical decision.

The fact that the effects of visually similar primes will be contingent upon the type of response that is required does have implications for our subsequent experiments. Since visual similarity produces large and opposite effects for word and nonword targets when there is an overt response to the prime, this means that we have a potentially sensitive measure of the availability of visual information when primes are presented in the parafovea and subjects are required to respond to those primes. However, since the utilization of visual similarity seems to be tied to very deliberate and prescribed processing of the prime in the lexical-decision task, a fair amount of skepticism would have to accompany any suggestion that similar effects obtained with parafoveal primes could be generalized to the normal reading process.

\section{Identical Primes}

When subjects were not required to respond to the prime (Group 1), word targets preceded by identical word primes were judged 129 msec faster than those preceded by the neutral primes $[\mathrm{t}(23)=6.19, \mathrm{p}<.001]$. It is not surprising that a prime that is both semantically and visually identical to a target is a more effective prime than one that is only semantically related or visually similar. Identical primes also produce large $(107-\mathrm{msec})$ benefits for nonword targets $[\mathrm{t}(23)=6.94, \mathrm{p}<.001]$.

Since word targets that are preceded by identical primes require two consecutive "yes" responses, the appropriate control requires subjects to respond "yes" to the Xs (Group 3). Having to make an overt response to the prime does not alter the fact that identical primes produce large benefits-viz., $66 \mathrm{msec}$ $[\mathrm{t}(23)=2.13, \mathrm{p}<.05]$.

The appropriate control for identical primes and nonword targets (a "no"-."no" response sequence) is the X-prime condition from Group 2. This comparison also shows large benefits-viz., $171 \mathrm{msec}$ $[\mathrm{t}(23)=8.26, \mathrm{p}<.001]$.

\section{EXPERIMENT 2}

The purpose of the second experiment was to determine if primes that begin five spaces to the right of fixation can produce semantic facilitation even when the subject's attention is directed toward an unrelated foveal prime and away from the semantically related parafoveal prime. This should closely simulate the typical allocation of attention that occurs during the reading of standard text, if one assumes that most of the reader's attention is directed toward recognizing the word currently projected to the fovea and updating the comprehension of the preceding text.

The spatial/temporal conditions of the experiment also simulate those encountered in normal reading. For example, in our identity condition, a foveal and parafoveal word are available for a brief period of time, after which the retinal position of the word initially projected to the left parafovea is displaced toward the fovea. This is equivalent to the case in 
which a reader saccades from the middle of one word to the middle of the next. In the present experiment, the foveal target was not presented until the subject responded to the unrelated foveal prime. Hence, the asynchrony between the onset of the word in the parafovea and its onset in the fovea was somewhat longer in this study $(500-600 \mathrm{msec})$ than in real reading (250-300 $\mathrm{msec})$. Although it would be desirable to have a more perfect match, we felt it was essential to use a task that permitted us to have control over the subject's attention to spatial location.

In summary, the attentional demands and the spatial-temporal pattern of stimuli reasonably simulate conditions that occur during normal reading. Furthermore, the task demands and display characteristics yield parafoveal primes that are very difficult to consciously recognize. Thus, the occurrence of semantic facilitation would strongly support the hypothesis that words presented in the parafovea can activate semantically related lexical entries without deliberate attention or conscious awareness.

\section{Method}

\section{Design}

There were sixteen within-subjects conditions formed by the combination of the four types of parafoveal primes (semantically related, visually similar, identical, or neutral), two types of foveal primes (unrelated words or unrelated nonwords), and two types of targets (word or nonword). Each letter string was presented only once to each subject, and the stimulus materials were counterbalanced in the same manner described for Experiment 1.

\section{Subjects}

Thirty-two students participated in the experiment as part of a class project in introductory perception.

\section{Procedure and Apparatus}

The procedure and apparatus used in this experiment were the same as those described for Experiment 1, with the following exceptions. First, there were two prime stimuli rather than one. The identical, semantically related, visually similar, and neutral primes used in the first experiment were presented in the parafovea at the same time that an unrelated prime was presented to the fovea. The foveal and parafoveal primes appeared on the screen immediately following the asterisk. The foveal prime was centered about the prior position of the asterisk, and the parafoveal prime began two character spaces to the right of the foveal prime. The separation between the point on the screen at which the asterisk appeared and the first letter of the parafoveal prime was $1.21 \mathrm{deg}$. The foveal and parafoveal primes remained on the screen for $133 \mathrm{msec}$. The subjects were instructed to look directly at the asterisk at the beginning of each trial. The brief exposure of the prime stimuli insured that the subjects looking directly at the asterisk would be unable to execute a saccade to the parafoveal prime while it still appeared on the screen.

The subjects were instructed to make lexical decisions about only the foveal prime and target. Although the relationship between the parafoveal primes and targets was explained, the subjects were instructed to ignore the letter strings that appeared to the right of the foveal primes. The subjects were easily able to follow this instruction and reported being aware of only a flash of light at the side of the screen. Error messages appeared after incorrect responses to the foveal prime or target. The target letter string was not presented if an error occurred on the foveal prime. The subjects exceeding an error rate of .06 were replaced.

\section{Stimuli}

The prime-target combinations tested in the first experiment were the same as the parafoveal prime-target combinations used in the second experiment. An additional set of words and nonwords that were both semantically and visually unrelated to the targets were selected to serve as the foveal primes. Each type of parafoveal prime (e.g., identical) was paired with an equal number of word and nonword foveal primes.

\section{Results and Discussion}

The mean response times for those targets that were responded to correctly were submitted to an analysis of variance treating parafoveal-prime type (identical, semantically related, visually similar, or neutral), foveal-prime type (word or nonword), and target type (word or nonword) as within-subjects variables. The only important result of this second experiment was that neither the main effect of parafoveal-prime type nor any interaction involving this factor was significant. The second panel of Table 1 shows the response times and error rates, collapsed across foveal-prime type, for word and nonword targets preceded by each of the four types of parafoveal primes.

It is clear that primes that produce large benefits when presented foveally do not produce similar benefits when presented to the parafovea in the context of a task that does not permit the subject to consciously attend to the information in the parafovea. In fact, inspection of Table 1 shows that the nonsignificant differences tend in the direction of costs, not benefits. These results seriously erode the strongest form of the hypothesis that was derived from Marcel's work, viz., that a word projected to the parafovea can activate its lexical representation and increase the accessibility of itself and other semantically related words, even when the subject is not consciously aware of the information in the parafovea and is allocating most of his resources toward processing information in the fovea.

\section{EXPERIMENT 3}

Our total failure to find any effects of either semantic or visual similarity in the second experiment led us to direct the subject's attention toward the parafoveal prime, in an attempt to see if we could produce patterns of facilitation with our lexical-decision task similar to those reported by Rayner et al. (1978) with a pronunciation task. In this experiment, the subjects were required to make lexical decisions about both the parafoveal prime and the target.

\section{Method}

\section{Design}

Each combination of the four types of parafoveal primes with the two types of targets and the two positions of the peripheral primes (right or left) produced 16 within-subjects conditions. 
Every word and nonword target appeared in all conditions across subjects, and each string of letters was presented only once to a subject.

\section{Subjects}

Thirty-two subjects who had not participated in either of the other two experiments volunteered for this experiment in order to partially satisfy an introductory psychology class requirement.

\section{Stimuli}

The parafoveal primes and targets used in the secpnd experiment were used again in the present experiment. The foveal prime in the third experiment was always a four-letter string of uppercase Ms.

\section{Procedure and Apparatus}

The procedure and apparatus used in this experiment were the same as those used in Experiments 1 and 2, except for the changes described. The foveal stimulus of upper case Ms appeared simultaneously with the parafoveal prime and remained in view for $133 \mathrm{msec}$. The string of Ms always appeared centered in the position in which the asterisk had been presented, and the parafoveal prime appeared two character spaces either to the right or left of the foveal Ms. The separation between the point at which the asterisk appeared and the first letter of the parafoveal prime on the right was $1.21 \mathrm{deg}$. When the parafoveal prime appeared to the left of the Ms, the separation between the asterisk and the last letter in the peripheral prime was $1.16 \mathrm{deg}$.

The subjects could not anticipate whether the parafoveal prime would appear to the right or left of the string of Ms, since presentation of the materials was randomized during each block of 32 trials. The subject was instructed to be sure to fixate the asterisk in the center of the screen. Two subjects who reported that they did not fixate the asterisk were replaced. The subjects were required to make lexical decisions about both the parafoveal prime and the target. An error message appeared on the screen only if an incorrect response was made to the target letter string. Since the parafoveal primes were very difficult to judge, the error message was omitted for mistakes on the prime. Unlike Experiments 1 and 2, targets were presented regardless of whether an error occurred on the prime decision. The subjects were replaced only if their error rates on the target decision were greater than .06 .

\section{Results and Discussion}

\section{Lexical-Decision Errors on the Parafoveal Prime}

In this experiment, the subject was required to make an initial lexical decision about the parafoveal prime. Since the nonword primes, excluding the neutral $\mathrm{X}$ primes, were all very similar to actual words, this decision should have been difficult when the nearest letter was $1.2 \mathrm{deg}$ from fixation. The overall error rate of .310 confirms this expectation.

Individual error rated for the first lexical decision were submitted to an analysis of variance treating target type (word or nonword), parafoveal-prime type (semantically related, visually similar, identical, or neutral), and parafoveal-prime location (left or right of fixation) as within-subjects variables. It is somewhat surprising that there was no right-visualfield advantage in the present experiment: neither the main effect of parafoveal-prime location nor any interaction involving this factor was significant. Consequently, Table 2 shows the error rates, collapsed across parafoveal-prime location, for the four types
Table 2

Error Rates for Lexical Decisions Made About Primes Presented to the Parafovea

\begin{tabular}{|c|c|c|c|c|c|}
\hline & \multirow[b]{2}{*}{ Target } & \multicolumn{4}{|c|}{ Prime Type } \\
\hline & & SR & VS & I & $\mathrm{Xs}$ \\
\hline & & \multicolumn{4}{|c|}{ Experiment 3} \\
\hline & $\begin{array}{l}\text { W } \\
\text { NW }\end{array}$ & $\begin{array}{l}.478 \\
.480\end{array}$ & $\begin{array}{l}.327 \\
.314\end{array}$ & $\begin{array}{l}.453 \\
.336\end{array}$ & $\begin{array}{l}.050 \\
.039\end{array}$ \\
\hline & & \multicolumn{4}{|c|}{ Experiment 4} \\
\hline Subject S.J. & $\begin{array}{l}\text { W } \\
\text { NW }\end{array}$ & $\begin{array}{l}.062 \\
.075\end{array}$ & $\begin{array}{l}.050 \\
.044\end{array}$ & $\begin{array}{l}.031 \\
.019\end{array}$ & $\begin{array}{l}.000 \\
.000\end{array}$ \\
\hline Subject S.N. & $\begin{array}{l}\text { W } \\
\text { NW }\end{array}$ & $\begin{array}{l}.106 \\
.133\end{array}$ & $\begin{array}{l}.222 \\
.109\end{array}$ & $\begin{array}{l}.146 \\
.127\end{array}$ & $\begin{array}{l}.031 \\
.068\end{array}$ \\
\hline
\end{tabular}

Note - Target $=$ target following prime. $S R=$ semantically related; $V S=$ visually similar; $I=$ identical; $X s=$ neutral $X s$.

of parafoveal primes that precede the word and nonword targets. It can be seen that the significant main effect of parafoveal-prime type $[F(3,93)=40.62$, $\mathrm{p}<.001]$ is mostly due to the extremely low error rates on the neutral Xs compared with the other types of parafoveal primes. No other main effects or interactions were significant.

\section{Lexical-Decision Times on the Targets}

Mean response times to targets that were judged correctly were submitted to an analysis of variance treating target type, parafoveal-prime type, and parafoveal-prime location as within-subjects factors. This analysis includes target latencies that were preceded by both correct and incorrect responses to the prime. The only significant findings were the main effect of target type $[F(1,31)=66.81, p<.001]$ and the Target Type by Parafoveal-Prime Type interaction $[F(3,93)=5.255, p<.05]$. The mean latencies and error rates for this interaction are shown in the third panel of Table 1. These means are collapsed across the location of the parafoveal prime since this variable had no effect.

Semantically related primes. Recall that the only difference between the present experiment and Group 2 of the first experiment is that the primes in the present experiment have been displaced to the parafovea. The same prime-target combinations that produced benefits of $53 \mathrm{msec}$ when the prime was presented foveally produced costs of $38 \mathrm{msec}$ when projected to the parafovea $[\mathrm{t}(31)=2.59, \mathrm{p}<.01]$. In direct contrast with Marcel's view, the costs obtained in the present experiment suggest that semantic facilitation may only occur when subjects can see and recognize the prime word, and that interference will occur when lexical access is misdirected by a degraded prime.

Identical primes. The same prime-target combinations that produced $66-\mathrm{msec}$ benefits for word targets 
and 171-msec benefits for nonword targets (Experiment 1, Group 2) produced no significant effects in this experiment. The nonsignificant trends are even in the wrong direction. Word targets preceded by identical primes were judged $5 \mathrm{msec}$ slower than those preceded by neutral primes. Similarly, nonword targets preceded by identical primes were judged 6 msec slower.

Visually similar primes. When subjects were required to make a lexical decision about visually similar primes presented foveally (Experiment 1, Group 2), there were $39 \mathrm{msec}$ of costs associated with word targets. Comparable results were obtained when the prime was moved to the parafovea, since word targets preceded by visually similar primes were judged $36 \mathrm{msec}$ slower than those preceded by the neutral primes $[\mathrm{t}(31)=2.24, \mathrm{p}<.05]$. In contrast, nonword targets that yielded benefits of $73 \mathrm{msec}$ when presented foveally show no effect (1 msec cost) in the parafovea.

We thought it might be informative to examine only the target latencies that followed a correct response to the prime. Although it's reasonable to expect a different pattern of results when subjects have more veridical information about the parafoveal prime, this additional analysis showed the same pattern of cost and benefit as the overall analysis. The small shifts in the magnitude of cost or benefit for specific prime-target combinations were usually nonsignificant and, in any event, theoretically unimportant.

\section{Summary}

Even when subjects are deliberately attending to parafoveal primes that begin about $1.2 \mathrm{deg}$ from fixation, there does not appear to be any support for the hypothesis that words presented parafoveally can consistently activate their lexical representations and facilitate subsequent judgments of semantically related words. This follows from the finding that word targets preceded by semantically related primes are judged slower, not faster, than those preceded by neutral primes.

It also appears to be the case that the effects of visual identity or similarity are either severely attenuated or eliminated entirely when the prime is shifted to the parafovea. The relative ineffectiveness of our identical primes appears to be at odds with the robust benefits reported by Rayner's group for identical primes preceding a target that was to be named as rapidly as possible. For example, the subject that received the neutral-asterisk control appears to show benefits for the identical primes of about $73 \mathrm{msec}$ at $1 \mathrm{deg}$ and $45 \mathrm{msec}$ at $3 \mathrm{deg}$ (estimated from Figure 2 of Rayner et al., 1978). Conceding the possibility that the contrast or graphics of our video monitor, or perhaps even the additional $.2 \mathrm{deg}$ of eccentricity, may have rendered our primes somewhat less identi- fiable than Rayner et al.'s (1978) 1-deg primes, it does not seem reasonable to assume that our primes were also more difficult to see than their 3-deg primes.

Rather than being due to stimulus quality, it occurred to us that the apparent discrepancy between Rayner et al.'s (1978) results and our own may be due to the fact that the three subjects tested in their pronunciation task were highly familiar with the stimulus materials, whereas the subjects in our lexical-decision task saw each prime and target only once. In addition to an unspecified number of preliminary trials, each subject in their study would have been exposed to each prime-target combination 12 times during the course of participating in both the standard eye movement condition and the simulation condition. This high degree of familiarity with the set of potential primes could provide a powerful supplement to the visual input during the perceptual synthesis of the parafoveal stimuli.

\section{EXPERIMENT 4}

In order to test the hypothesis that familiarity and/or expectancy plays a significant role in determining the effectiveness of parafoveal primes, we decided to replicate Experiment 3 with two highly practiced subjects. One subject, the investigator S.N., was intimately familiar with the materials, since she selected them, entered them into the microcomputer, and suffered the consequences of whatever incidental learning accompanies the running of innumerable subjects. The other subject, S.J., was a research assistant who had also received a large amount of passive exposure to the materials during the course of data collection. In addition to possessing this preexperimental knowledge, both subjects practiced on those trials that would have been equivalent to the entire experiment for four subjects in Experiment 3. The experimental sessions for each of the subjects in Experiment 4 were the equivalent of those for eight subjects from Experiment 3. Although the subjects were very familiar with the potential set of parafoveal primes, the on-line randomization procedures insured that they could not anticipate in advance" which particular prime might occur on the next trial.

\section{Results and Discussion}

A high degree of familiarity with the materials produced a rather remarkable improvement in the initial lexical decision. Subjects S.N. and S.J. had error rates of .12 and .04 compared with the average error rate of .31 for the 32 native subjects in Experiment 3. Error rates for the individual subjects in the present experiment for each type of parafoveal prime are shown in the bottom panel of Table 2 and may be compared with the group averages obtained in the third experiment. 
The bottom portion of Table 1 shows the individual latencies and error rates on the word and nonword targets for each of the parafoveal-prime types. It is clear that familiarity had a dramatic effect on the priming capabilities of the parafoveal primes. On the average, the two highly practiced subjects showed $265 \mathrm{msec}$ of benefits for identical primes and $172 \mathrm{msec}$ for semantically related primes as compared with 5 and $38 \mathrm{msec}$ of costs for the 32 naive subjects who participated in the third experiment. The highly practiced subjects even showed the bias effects on visually similar primes that were observed in the two groups from Experiment 1 that were required to make lexical decisions about both a foveal prime and the target. Since all of the visually similar primes were nonwords, the initial response for these trials was always "no." When the target was a nonword and required another "no" response, visual similarity produced mean benefits of $44 \mathrm{msec}$; but when the target was a word and, therefore, required the opposite response, visual similarity produced average costs of $30 \mathrm{msec}$.

One might criticize the importance of Experiment 4 by suggesting that our sophisticated subjects were so familiar with the particular prime-target pairs that they frequently knew what target would follow (and, therefore, its lexical status) once they had identified the prime. Neither subject reported engaging in such a strategy, and, in fact, during data collection, both subjects expressed some doubt as to whether they were producing any priming effects at all. Furthermore, deliberate response priming on the basis of learned associations between parafoveal prime and target should have benefited not only the identical and semantically related primes, but also the visually similar primes. In contrast with this expectation, significant costs were observed when a visually similar prime (e.g., oqqn) preceded a word target (e.g., eggs). In summary, when subjects are highly practiced and familiar with the potential set of parafoveal primes, semantic and visual priming effects that are comparable to or larger than those reported for practiced subjects in Rayner's (1975) naming task are obtained.

\section{CONCLUSIONS}

The goal of this research was to shed some light on the contributions of parafoveal processing in reading. More specifically, we asked if it would be reasonable to entertain the working hypothesis that the visual and/or lexical processing of upcoming words in the parafovea will speed the reading process when an eye movement brings that part of the text into the fovea. Since consistent priming effects have been obtained only with subjects who are highly practiced on the same set of stimulus materials, it is tempting to answer our question by replying that parafoveal processing is likely to contribute to reading efficiency only when readers are enjoying a favorite novel for the second or third time. However, this conclusion ignores the fact that expectations about the next word are frequently generated by contextual redundancies, regardless of whether or not the reader is already familiar with the specific passage in hand. Accordingly, an interesting and potentially valuable extrapolation from our data to normal reading would suggest that gains in reading efficiency accrue from the preprocessing of anticipated words in the parafovea, but not for those words that have little or misleading contextual constraint.

A recent study by $\mathrm{McClelland}$ and $\mathrm{O}^{\prime}$ Regan (in press) provides further support for such a model. In two experiments, subjects named foveal word targets that were preceded by parafoveal primes that were either identical to the target, visually similar (same shape and same end letters) to the target, or neutral. In the first experiment, the target set was either constrained (8 targets repeated 49 times each) or unconstrained (392 targets presented only once each). Large benefits were derived from the identical and visually similar primes in the constrained condition. In contrast, the unconstrained condition yielded smaller benefits for the identical primes and nonsignificant costs for the visually similar primes. The manipulation and pattern of results are very similar to our comparison between naive (Experiment 3) and highly practiced (Experiment 4) subjects-viz., constraints generated by familiarity with the materials allowed much greater benefit to be derived from parafoveal primes. However, it should be noted that identical primes produced no benefits with our naive subjects, as compared with small, but significant, benefits in the unconstrained condition of McClelland and $O$ 'Regan. A likely reason for this is that our primes began five character spaces $(1.2 \mathrm{deg})$ from the fixation, whereas their primes were only 2.5 spaces $(.8 \mathrm{deg})$ from the center of the fovea.

The second experiment reported by McClelland and $O ' R e g a n$ used a preceding sentence that provided strong, weak, or no constraint for the target. The benefits gained from the parafoveal prime increased with the strength of the context. Most provocative were the results obtained with low-constraint sentences and visually similar primes. Alone, neither of these sources of information are sufficient to facilitate performance. However, together they produce significant benefits.

The priming studies that are reported and reviewed support the conclusion that readers can benefit little from the preprocessing of visual information in the parafovea unless it can be combined with expectations derived from the preceding context. However, this conclusion must be evaluated in the context of the known effects of perturbations of parafoveal 
material on eye movements during normal reading. Rayner's 1975 report is typical of the method used and findings obtained with this paradigm. The reader's eye movements were monitored, and certain critical words were altered during saccades that were launched at various distances before the critical-word location. If one assumes that the reading process will be disrupted whenever the reader detects a difference between what is preprocessed in the parafovea and what is found when that part of the text is finally brought into the fovea, then one can use average fixation duration to infer the types of information that are available in the parafovea. On the basis of this evidence, Rayner concluded that readers can pick up visual characteristics, such as word shape and end letters, as far as 7-12 spaces from fixation.

We would like to raise two separate issues with respect to the relationship between the eye movement data and the priming results obtained with either the lexical-decision task or the naming task. First, the amount of semantic and syntactic redundancy in the test paragraphs used by Rayner may have provided enough constraint to generate expectations for the critical words. Since Zola (Note 1) has shown that fixation durations for critical words are shorter in highly constrained contexts, it appears to be the case that fixation duration is sensitive to preceding context in a manner similar to that found for reaction time in lexical-decision or naming tasks. Accordingly, differences in fixation duration produced by moderate, slight, or no changes at the critical-word location could be predicted by the model described above. That is, in the presence of adequate contextual constraint, preprocessing of parafoveal information should lead to shorter fixation durations to the degree that the visual information in the parafovea matches that which is eventually brought into the fovea.

A second issue concerns the relative merits of the evidence obtained through measurements of interference in fixation duration vs. measurements of facilitation in a priming paradigm. The fixation-duration measure has one obvious advantage in that it can be used during reading, the very process we want to study. Its primary drawback is that one must rely on relative degrees of interference in order to make inferences about possible sources of facilitation during normal reading. The specific hypothesis under investigation has been whether or not preprocessing of parafoveal information can speed the reading process when an eye movement brings the upcoming text into foveal view. It is a nontrivial leap of faith to assume that parafoveal information that is capable of producing disruption (i.e., longer fixation durations) when varied will have a beneficial effect when the text is not perturbated. In fact, the cost-benefit analysis available in the priming paradigms used by McClelland and O'Regan and in the present studies makes this danger readily apparent. In the absence of any contextual constraint, a departure from per- fect identity will sometimes produce costs and will never produce benefits.

Thus, both the reaction-time measure used in the priming paradigms and the fixation-duration measure used in normal reading indicate that readers are sensitive to a variety of visual features present in the parafovea. However, only the priming studies can be used to determine the conditions that permit the reader to derive benefit from the information available in the parafovea. The clear implication from these priming studies is that readers benefit from the preprocessing of parafoveal information only when this visual information is supplemented by an adequate level of contextual constraint. Complete confidence in this conclusion awaits additional research that uses both the cost-kenefit analysis used in the priming paradigms and a task more similar to natural reading.

\section{REFERENCE NOTE}

1. Zola, D. The perception of words in reading. Paper presented at the meetings of the Psychonomic Society, Phoenix, November 1979.

\section{REFERENCES}

Antos, S. J. Processing facilitation in a lexical decision task. Journal of Experimental Psychology: Human Perception and Performance, 1979, 5, 527-545.

Clark, H. H. The language-as-fixed-effect fallacy: A critique of language statistics in psychological research. Journal of Verbal Learning and Verbal Behavior, 1973, 12, 335-359.

GEYER, L. H. Recognition and confusion of the lowercase alphabet. Perception \& Psychophysics, 1977, 22, 487-490.

MARCEL, T. Unconscious reading: Experiments on people who do not know that they are reading. Visible Language, 1978, 12, 391-404.

McClelland, J. L., \& O'Regan, J. K. The role of expectations in the use of parafoveal visual information in reading. Journal of Experimental Psychology: Human Perception and Performance, 1981, 7(3), in press.

Meyer, D. E., Schvaneveldt, R. W., \& Ruddy, M. G. Loci of contextual effects on visual word-recognition. In P. M. A. Rabbitt (Ed.), Attention and performance VI. New York: Academic Press, 1975.

Neely, J. H. Semantic priming and retrieval from lexical memory: Evidence for facilitatory and inhibitory processes. Memory \& Cognition, 1976, 4, 648-654.

Posner, M. I., \& Snyder, C. R. Facilitation and inhibition in the processing of signals. In P. M. A. Rabbitt \& S. Dornic (Eds.), Attention and performance $V$. New York: Academic Press, 1975.

RAYNER, K. The perceptual span and peripheral cues in reading. Cognitive Psychology, 1975, 7, 65-81.

Rayner, K., McConkie, G. W., \& Ehrlich, S. Eye movements integrating information across fixation. Journal of Experimental Psychology: Human Perception and Performance, 1978, 4, 529-544.

Rayner, K., McConkie, G. W., \& Zola, D. Integrating information across eye movements. Cognitive Psychology, 1980, 12, 206-226.

Schvaneveldt, R. W., \& McDonald, J. E. Semantic context and the encoding of words: Evidence for two modes of stimulus analysis. Journal of Experimental Psychology: Human Perception and Performance, 1981, 7(3), in press.

(Received for publication October 29, 1980; revision accepted February 20, 1981.) 